\section{Computer-Aided Tinnitus Detection based on Brain Network Analysis of EEG Functional Connectivity}

\author{
Mohagheghian F. ${ }^{10}$, Makkiabadi B..$^{2,3}$, Jalilvand H.4, \\ Khajehpoor H.5,6, Samadzadehaghdam N.5,6, Eqlimi E.,6, \\ Deevband M. R. ${ }^{1 * \odot() ~}$
}

\begin{abstract}
Background: Tinnitus known as a central nervous system disorder is correlated with specific oscillatory activities within auditory and non-auditory brain areas.

Several studies in the past few years have revealed that in the most tinnitus cases, the response pattern of neurons in auditory system is changed due to auditory deafferentation, which leads to variation and disruption of the brain networks.

Objective: In this paper, we introduce an approach to automatically distinguish tinnitus individuals from healthy controls based on whole-brain functional connectivity and network analysis.

Material and Methods: The functional connectivity analysis was applied to the resting state electroencephalographic (EEG) data of both groups using Weighted Phase Lag Index (WPLI) for various frequency bands in 2-44 Hz frequency range. In this case- control study, the classification was performed on graph theoretical measures using support vector machine (SVM) as a robust classification method.
\end{abstract}

Results: Experimental results showed promising classification performance with a high accuracy, sensitivity, and specificity in all frequency bands, specifically in the beta2 frequency band.

Conclusion: The current study provides substantial evidence that tinnitus network can be successfully detected by consistent measures of the brain networks based on EEG functional connectivity.

Citation: Mohagheghian F, Makkiabadi B, Jalilvand H, Khajehpoor H, Samadzadehaghdam N, Eqlimi E, Deevband M. R. ComputerAided Tinnitus Detection based on Brain Network Analysis of EEG Functional Connectivity. J Biomed Phys Eng. 2019;9(6):687-698. https://doi.org/10.31661/jbpe.v0i0.937.

\section{Keywords}

Tinnitus; Electroencephalography; Network Aanalysis; Functional Connectivity; Classification

\section{Introduction}

$\bigcirc$ ubjective tinnitus is defined as an internally generated auditory phantom percept in the absence of any external auditory stimuli [1-3]. It is a prevalent disorder; about $5-15 \%$ of the population of the western societies suffer from chronic tinnitus and the quality of life of $1-3 \%$ of the population is influenced by severe tinnitus, which is accompanied by depression in $50 \%$, and by insomnia in $40 \%$ of the cases [4-6]. Hearing impairment is experienced in approximately $80 \%$ of all people with tinnitus, and in these cases, the frequency range of hearing loss corresponds to the frequency spectrum of the phantom sound [7, 8]. Nevertheless, approximately $25 \%$ of individuals with tinnitus have normal hearing thresholds. On the other hand, a hyperacusis disorder,
${ }^{1} \mathrm{PhD}$, Department of Medical Physics and Biomedi-

cal engineering, School of

Medicine, Shahid Beheshti

University of Medical Sci-

ences (SBMU), Tehran, Iran

${ }^{2} \mathrm{PhD}$, Department of Medi-

cal Physics and Biomedi-

cal engineering, School of

Medicine, Tehran Univer-

sity of Medical Sciences

(TUMS), Tehran, Iran

${ }^{3} \mathrm{PhD}$, Research Center for

Biomedical Technology and

Robotics (RCBTR), Institute

of Advanced Medical Tech-

nologies (IAMT), Tehran

University of Medical Sci-

ences (TUMS), Tehran, Iran

${ }^{4} \mathrm{PhD}$, Department of Audi-

ology, School of Rehabilita-

tion, Shahid Beheshti Uni-

versity of Medical Sciences

(SBMU), Tehran, Iran

${ }^{5} \mathrm{MSc}$, Department of Medi-

cal Physics and Biomedi-

cal engineering, School of

Medicine, Tehran Univer-

sity of Medical Sciences

(TUMS), Tehran, Iran

${ }^{6} \mathrm{MSc}$, Research Center for

Biomedical Technology and

Robotics (RCBTR), Institute

of Advanced Medical Tech-

nologies (IAMT), Tehran

University of Medical Sci-

ences (TUMS), Tehran, Iran

*Corresponding author: M. R. Deevband

Department of Medical

Physics and Biomedi-

cal engineering, School

of Medicine, Shahid

Beheshti University

of Medical Sciences

(SBMU), Tehran, Iran

E-mail: mdeevband@

sbmu.ac.ir

Received: 12 May 2018

Accepted: 30 June 2018 
which is defined as an increased sensitivity to the sensed sounds, is frequently experienced by people with tinnitus.

While tinnitus is associated with a complex pathophysiology and its underlying neuronal mechanisms is highly challenging and unknown, it is widely accepted that the auditory deafferentation is the most frequent etiology of tinnitus, regardless of having hearing impairment [9]. Several researches show that tinnitus is accompanied with spontaneous variations in auditory and non-auditory regions [10].

According to neuroimaging studies, the human brain is assumed as an organization with the different degrees of the small-worldness [11-14]. Such organization is able to optimize the functional integration and segregation [15] and therefore efficiently transfer the information among its different pairs of nodes $[16,17]$. A number of previous studies have investigated the topological alteration of the brain network and the reduction of the smallworldness in brain-related pathologies. These changes were led to reduce the proficiency of transferring the information in global and local brain regions reported in disorders such as Schizophrenia, Parkinson, and Alzheimer [18]. Tinnitus network was investigated in [19] to identify variation of topological patterns in comparison with healthy individuals. The authors found distinct properties of connectivity networks in tinnitus and healthy subjects and hypothesized that correlated uncertainty with auditory deafferentation is led to change the topology of tinnitus network.

In the present paper, we attempt to assess the feasibility of tinnitus detection via topological properties of the brain networks. The main contribution of this work is automatically distinguishing healthy and tinnitus subjects based on the changes of graph-theory-based metrics extracted from functional connectivity of sensor space EEG data. In the next section, the EEG data acquisition, processing approaches, and network analysis methods are briefly outlined. The results of connectivity and network analysis are presented in Section 3. In section 4 , the conclusion of the results, discussion, and limitations of the work as well as the suggestion for future studies is presented.

\section{Material and Methods}

\section{Participants}

In this case- control study, eight participants with tinnitus (two females; mean age: 48.2 years; range: 28-59) and eight healthy individuals (three females; mean age: 30.8 years; range: 25-42) were included in the study. All individuals with tinnitus suffered from chronic tinnitus for duration of at least 12 months (range: 1-30 years). Our exclusion criteria were pulsatile tinnitus, Meniere's disease, seizure, physical disability and neurological disorders, e.g. brain tumors. None of the subjects in control group has suffered from tinnitus. All subjects were given written informed consent and informed about the aims of the experiment before it. Extra written consent in accordance with the Ethical Committee of the Sahid Beheshti University of Medical Sciences (SBMU) was given to individuals with tinnitus. All subjects from two groups are reported to be right-handed. To assess the severity of a perceived tinnitus handicap, the standardized Persian version of Tinnitus Handicap Inventory (THI) questionnaire was presented to the participants with tinnitus. A tinnitus handicap is clustered in five categories, including slight (0-16), mild (18-36), moderate (38-56), severe (58-76) and catastrophic (78-100) [20]. In our study, one participant was characterized by a slight, mild moderate, five ones and one catastrophic handicap.

\section{EEG Data Acquisition}

Three 5-minute eyes open resting state EEG data were recorded from tinnitus participants. The EEG recordings of healthy individuals obtained as the $1^{\text {st }}$ Iranian BCI Competition (iBCIC) were held in National Brain Mapping Laboratory (NBML) (http://nbml.ir/) during 
2-minute eyes open resting state. All participants were instructed to pay attention to a neutral color background in the front screen for the duration of recording and attempt not to think about anything in particular during the whole experiment. All EEG data were recorded using a 64-channel g.tec (http://www.gtec.at/) EEG system (g. HIamp) in NBML. The reference channel was placed on right ear lobes for all individuals. The sampling frequency of 1200 $\mathrm{Hz}$ was selected for tinnitus group and 2400 $\mathrm{Hz}$ for controls. The sampling frequency of controls was resampled to $1200 \mathrm{~Hz}$ in a preprocessing step.

\section{Data Processing}

EEG data were preprocessed using EEGLAB [21] and Fieldtrip [22] toolboxes for MATLAB (MATLAB R2016a, The MathWorks, Inc, Natick, Massachusetts, United States). High-pass (the cut-off frequency of $2 \mathrm{~Hz}$ ) and band-stop filters (to exclude $50 \mathrm{~Hz}$ power line noise and its harmonic frequencies) were applied to the raw data. Re-referencing the data to the common average reference and manually rejecting the artifacts by visual inspections were accomplished using EEGLAB. The independent component analysis (ICA) was employed to remove artifactual components (e.g. eye blinks, eye movements, heartbeat, and muscle artifacts). The preprocessed data, containing the least amount of artifacts, was segmented into 18 trials of 5-second (90 seconds) when the whole time was in the range of previous resting-state studies [23, 24]. Fieldtrip was utilized for further processing of the EEG data in order to perform the connectivity analysis. Graph-based network measures were generated by using Brain Connectivity Toolbox (BCT) [25] from the extracted connectivity matrices.

\section{The Functional Connectivity Analy- sis}

The functional connectivity was computed between all pairwise electrodes (sensor space).
The connectivity values were calculated for each eight-frequency band based on previous researches in tinnitus [19, 26, 27] including delta $(2-3.5 \mathrm{~Hz})$, theta $(4-7.5 \mathrm{~Hz})$, alpha1 (8$10 \mathrm{~Hz})$, alpha2 (10-12 Hz), beta1 (13-18 Hz), beta2 $(18.5-21 \mathrm{~Hz})$, beta3 $(21.5-30 \mathrm{~Hz})$ and gamma $(30.5-44 \mathrm{~Hz})$. Graph theory analysis was applied to the eight $63 \times 63$ connectivity matrices, which was computed for each subject.

Weighted Phase Lag Index (WPLI) Connectivity Analysis

WPLI connectivity measure was primarily introduced by [28] as an extension to the Phase Lag Index (PLI). WPLI estimates the phase leads and lags between two interacted time series (i.e. the electrode or source signals). A great value of WPLI connectivity would be obtained in the specified frequency when two signals have high functional coherence [29]. The imaginary part of the cross-spectrum applied in WPLI leads to reduce the influence of common sources of noise (e.g. heartbeat) and variations in the synchronization phase. Furthermore, WPLI is highly sensitive to properly detect phase interactions of spatially close signals and shows robustness to volume conduction $[28,30,31]$.

$W P L I_{x y}=\frac{n^{-1} \sum_{t=1}^{n}\left|\operatorname{imag}\left(S_{x y t}\right)\right| \operatorname{sgn}\left(\operatorname{imag}\left(S_{x y t}\right)\right)}{n^{-1} \sum_{t=1}^{n}\left|\operatorname{imag}\left(S_{x y t}\right)\right|}$

where $S_{x y t}$ is the cross-spectrum of the timeseries $x$ and $y$ at the time point t, and sgn is the sign function. Function imag(.) returns only the imaginary component of the cross-spectrum

\section{SVM Classification Approach}

A support vector machine was adopted to classify the tinnitus group from healthy controls. SVM works vigorously with a small number of training samples and a large number of features [32]. As the labeled samples are used for training, SVM is a supervised classifier, which can be applied with several kernels 
such as linear, polynomial, Multilayer Perceptron (MLP) and radial basis function (RBF) kernel [33-35]. The specified network measures were extracted from connectivity matrices as classification features. A combination of feature ranking and Principal Component Analysis (PCA) was applied to the features to provide the feature set with a minimum redundancy. Generally, PCA known as a classical approach achieves optimum linear transform to generate the mutually uncorrelated features. Firstly, all features normalized using linear scaling to the unit range, which transformed the feature vector to be in the range of 0 and 1. Subsequently, the entropy-based approach was used for feature ranking. In our study, we selected the 60 top ranked features to yield the best classification performance with a reasonable running time. Finally, the new feature vector was produced by keeping the first few principal components of PCA. Non-linear SVM classification with RBF kernel was accomplished. Owing to the limited number of samples, Leave-One-Out Cross-Validation (LOOCV) strategy was used to evaluate the classification performance.

\section{Network Analysis}

Brain networks are constructed by the calculation of functional couplings between different regions of the brain. Complex network analysis quantifies the network topologies with a few neurobiologically relevant measures to characterize the fundamental traits of the complex systems. Brain as a complex network might be notably characterized by the complex network analysis. [25]. A network can be mathematically represented by a set of nodes and links between node pairs. Node strength is the sum of the weights of links or edges connected to a node.

$$
k_{i}=\sum_{j \in N} w_{i j}
$$

where $N$ is the set of all nodes in the network and the links $(i, j)$ are related by the connection weight $w_{i j}$

The shortest weighted path length between two nodes $i$ and $j$ is determined by:

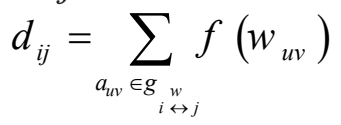

where $f$ is a map (e.g. an inverse) from the

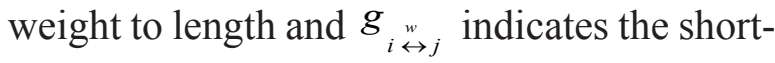

est weighted path between nodes $i$ and $j$.

The averaged shortest path length between all the node pairs in a network is known as the characteristic path length and is inversely related to the global efficiency of the network $[12,25]$. The characteristic path length [16] of the network is defined as:

$$
L=\frac{1}{n} \sum_{i \in N} \frac{\sum_{j \in N, j \neq i} d_{i j}}{n-1}
$$

where $n$ shows the number of nodes.

The global efficiency [36] of the network is characterized by:

$$
E=\frac{1}{n} \sum_{i \in N} \frac{\sum_{j \in N, j \neq i} d_{i j}^{-1}}{n-1}
$$

The number of triangles around a node $i$ is defined as a basis for measuring segregation:

$$
t_{i}=\frac{1}{2} \sum_{j, h \in N} a_{i j} a_{i h} a_{j h}
$$

where $a_{i j}$ indicates the status of the connection between nodes $i$ and $j: a_{i j}=1$ when the link $(i, j)$ exists; $a_{i j}=0$ when there is no connection between $i$ and $j\left(a_{i j}=1\right.$ for all $i$ ) [25].

A clustering coefficient reflects the degree that the connected nodes in a graph tend to form clusters and can illustrate the degree of local connectivity in the network $[12,16]$. The clustering coefficient of the network is described by:

$$
C=\frac{1}{n} \sum_{i \in N} \frac{2 t_{i}}{k_{i}\left(k_{i}-1\right)}
$$

Local efficiency of the network [36] is calculated by: 


$$
E_{l o c}=\frac{1}{n} \sum_{i \in N} \frac{\sum_{j, h \in N, j \neq i} a_{i j} a_{i h}\left[d_{j h}\left(N_{i}\right)\right]^{-1}}{k_{i}\left(k_{i}-1\right)}
$$

where $d_{j h}\left(N_{i}\right)$ is the length of the shortest path between $j$ and $h$, that contains only neighbors of $i$.

The global efficiency and characteristic path length measure the integration of the network, while the clustering coefficient and local efficiency are measures for the network functional segregation. Complex networks, characterized with the small-worldness [37], are defined as the networks with the great clustering coefficient and low characteristic path length.

$$
S=\frac{C / C_{\text {rand }}}{L / L_{\text {rand }}}
$$

where $C$ and $C_{\text {rand }}$ are the clustering coefficients, and $L$ and $L_{\text {rand }}$ are the characteristic path lengths of the tested network and a random network, respectively.

Accordingly, the small world organizations have simultaneously notably segregated and integrated topologies $[15,25]$. It has been previously reported that neuropathological disorders cause the change of the small-worldness with a reduction in global integration [38]. In this study, some neurobiological measures of the brain networks were characterized to extract the functional integration and segregation of the brain regions.

\section{Results}

The WPLI connectivity metric was determined between the pairwise electrode signals for both tinnitus and control groups at eight frequency bands using Brain Connectivity Toolbox for MATLAB.

Figure 1 represents the variation of averaged WPLI connectivity values as a function of frequency in control and tinnitus groups. Principally, higher values of averaged WPLI for the tinnitus group are observed in $2-3.5 \mathrm{~Hz}$, 4-5.2 Hz and 9-10 Hz considered in the range of delta, theta, and alpha1 frequency bands, respectively. In addition, the figure indicates the highest connectivity values in the alpha band for both groups. The observed WPLI peak

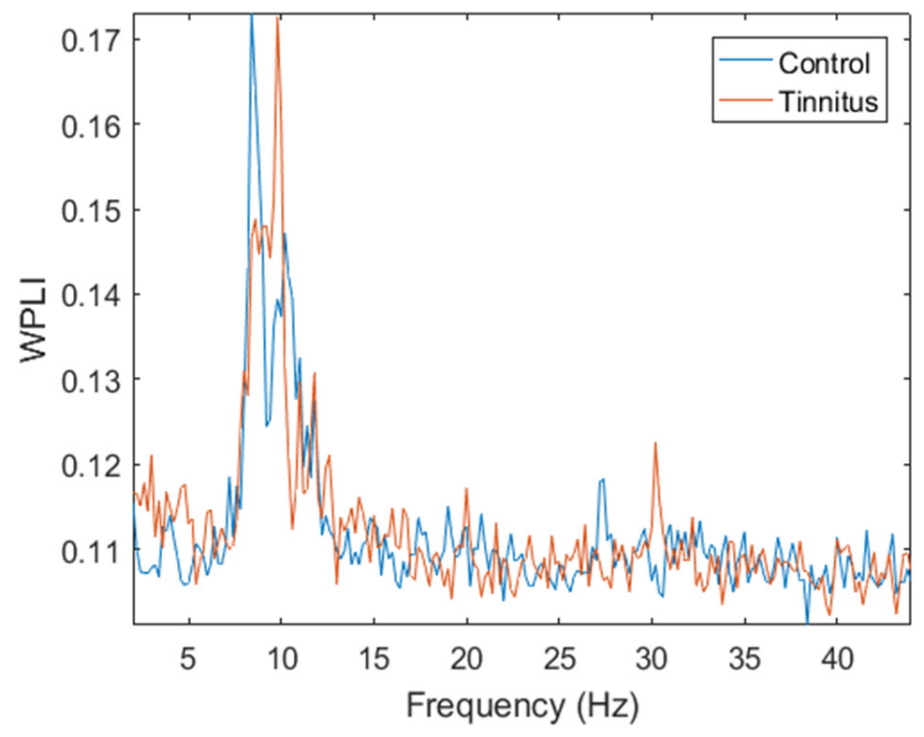

Figure 1: Variation of averaged WPLI connectivity as a function of frequency for tinnitus and control groups. 
in the alpha frequency range for both groups might be related to power values, which become maximum in the alpha band.
Figure 2 indicates the averaged WPLI matrices are calculated between the pairwise combination of electrode signals for control
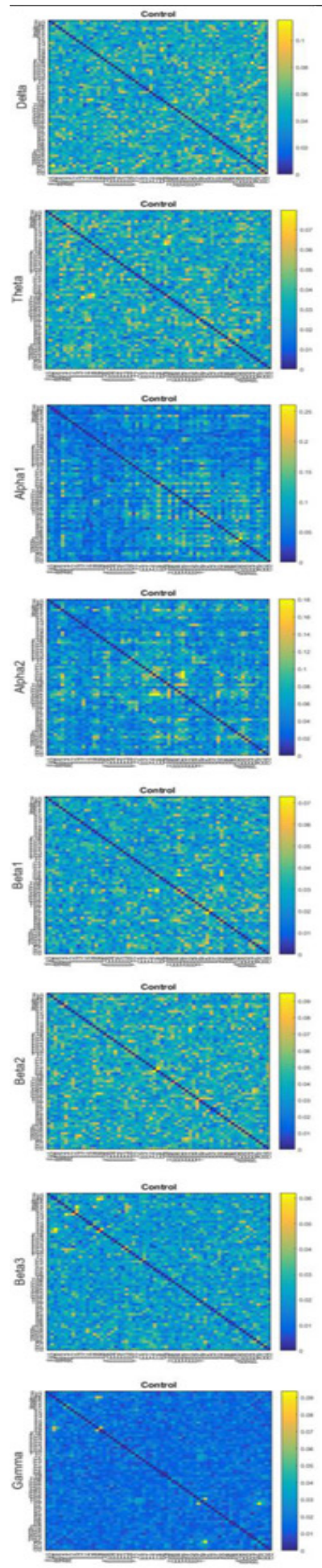
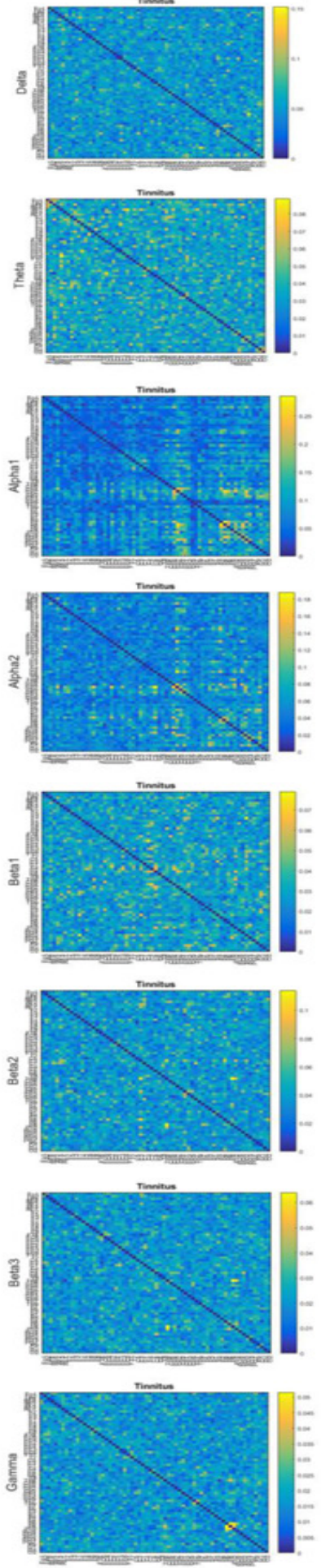
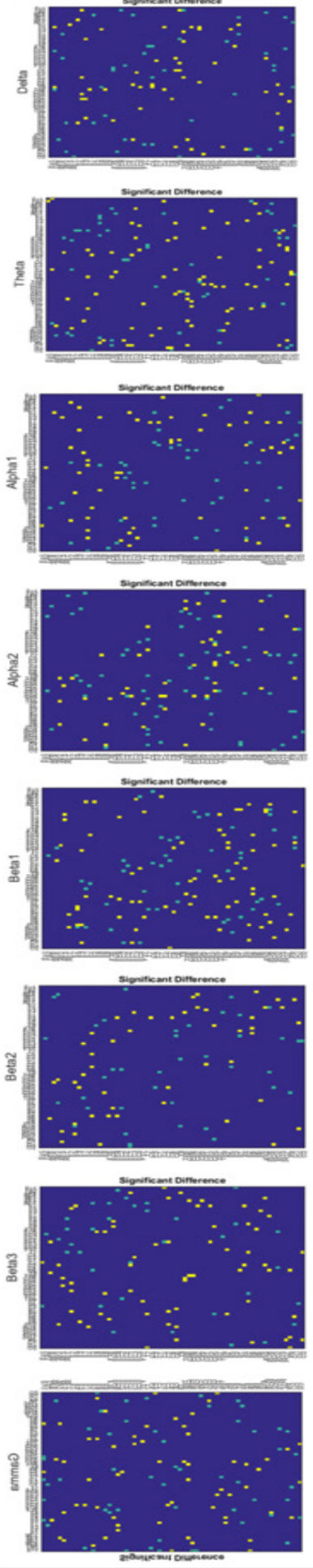

Figure 2: Averaged WPLI connectivity strength matrices in 8 frequency bands, left: healthy controls, middle: tinnitus, right: significant difference matrices, representing Wilcoxon signed-rank test for the significance of any difference across two groups for all connections. 
and tinnitus groups in each frequency band. Furthermore, the significant differences in the calculated functional connectivity between tinnitus and controls are presented in the figure (right column). In the right column the yellow pixels correspond to node pairs with the significantly higher WPLI connectivity in tinnitus compared to controls, the light blue pixels correspond to node pairs with the significantly greater connectivity in controls relative to tinnitus, and the dark blue areas show node pairs with no significant difference of functional connectivity between controls and tinnitus group.

According to the significant differences $(p<0.05)$, the electrodes T7 (middle temporal) and CP5 (centroparietal) displayed relatively the most couplings with other electrodes in the alpha 2 frequency domain. Further, the Fz (midline frontal) and the FP1 electrode (anteriofrontal) indicated a large number of connections with other electrodes in the alpha1 and gamma frequency bands, respectively. In addition, the significant differences implied that the delta, theta, and gamma bands showed stronger averaged WPLI for tinnitus group, while greater averaged WPLI was recognized in the alpha and beta bands for controls.

Figure 3 indicates four brain network measures calculated based on WPLI connectivity matrices in different frequency bands for both groups: the node strength, clustering coefficient, local efficiency and characteristic path length. As the distribution of these measures did not have a normal distribution, Wilcoxon signed-rank test was performed to investigate the main effect of groups (tinnitus and control) for each of the measures. The Wilcoxon signed-rank test expressed a significant effect of groups in some frequency bands $(p<0.05)$. Figure 3.a illustrates that the averaged node strength of control group was significantly stronger than the tinnitus subjects in the alpha 2 , beta 2 and beta 3 frequency bands $(p<0.05)$, while in the delta, theta, and beta 1 frequency domains the tinnitus network had significantly greater averaged node strength than the controls $(\mathrm{p}<0.05)$. There were no significant effects for the alphal $(p=0.7528)$ and gamma $(p=0.9672)$ frequency bands.

The averaged clustering coefficients of two groups are shown in Figure 3b. The averaged clustering coefficient of tinnitus group was significantly larger than controls in the alpha2, beta 2 , and beta 3 frequency bands $(p<0.05)$, while the healthy control group showed significantly larger averaged clustering coefficient than tinnitus in the delta, theta, and alphal frequency domains $(p<0.05)$. No significant effect was observed for the betal $(p=0.1819)$ and gamma $(p=0.7632)$ frequency bands.

The averaged local efficiency of two groups, presented in Figure 3c demonstrates that for tinnitus network this measure was significantly larger than healthy controls in the alpha2, beta 2 and beta3 frequency bands $(p<0.05)$. The averaged local efficiency of controls was significantly greater than tinnitus network in the theta and alphal frequency bands $(p<0.05)$. No significant effects were observed for the delta $(p=0.5378)$, betal $(p=0.9618)$ and gamma $(p=0.8426)$ frequency bands.

Figure $3 \mathrm{~d}$ displays the averaged characteristic path length of two groups. The averaged characteristic path length of tinnitus group was significantly greater than controls in the alpha2, beta 2 and beta 3 frequency bands $(p<0.05)$. The healthy controls showed significantly higher averaged clustering coefficients than tinnitus in theta and betal frequency domains $(p<0.05)$. No significant effects were observed for the delta $(p=0.1564)$, alphal $(p$ $=0.2417)$ and gamma $(p=0.1429)$ frequency bands.

Table 1 demonstrates the results of the SVM classifier using RBF kernel. Four brain network measures including node strength, clustering coefficients, local efficiency and characteristic path length were applied as the feature vector in each frequency band. In order to quantify the classifier performance accuracy, sensitivity and specificity of the classification 


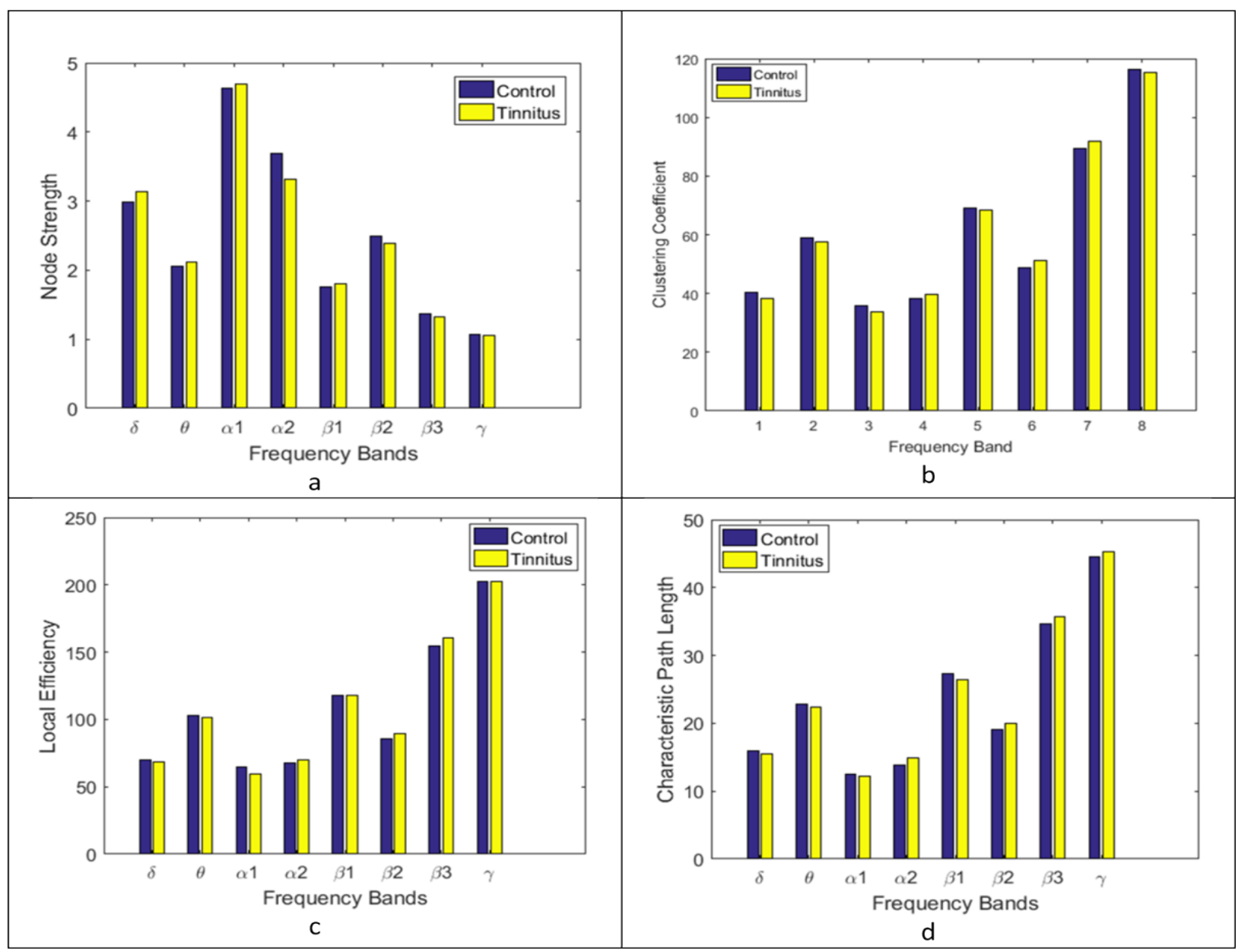

Figure 3: Averaged network measures: a. node strength, b. clustering coefficient, c. local efficiency and $d$. characteristic path length.

were computed. The maximum classification accuracy of $91.7 \%$ was identified in the delta, alpha1, beta1, and beta 2 frequency bands. The high classification accuracy of $91.7 \%$ was observed in delta, alpha1, and batal frequency bands when beta2 shows the highest value of $100 \%$. The maximum sensitivity of $100 \%$ was obtained in the beta 1 and beta 2 frequency bands; and the specificity of $100 \%$ in the delta, alpha1, and beta 2 frequency domains. The relatively low accuracy of $75 \%$ was recognized in the alpha 2 and beta 3 bands. The lowest sensitivity and specificity of $66.7 \%$ were observed in the alpha 2 and beta 3 frequency bands, respectively. All accuracy, sensitivity, and specificity were $100 \%$ in the beta 2 fre-
Table 1: classification results of SVM in eight different frequency bands.

Frequen- Accuracy Sensitiv- Specificcy bands $\quad(\%) \quad$ ity (\%) ity (\%)

\begin{tabular}{cccc}
\hline Delta & 91.7 & 83.3 & 100 \\
\hline Theta & 83.3 & 83.3 & 83.3 \\
\hline Alpha1 & 91.7 & 83.3 & 100 \\
\hline Alpha2 & 75.0 & 66.7 & 83.3 \\
\hline Beta1 & 91.7 & 100 & 83.3 \\
\hline Beta2 & 100 & 100 & 100 \\
\hline Beta3 & 75.0 & 83.3 & 66.7 \\
\hline Gamma & 83.3 & 83.3 & 83.3
\end{tabular}


quency band.

\section{Discussion}

In this study, resting state EEG data were explored to distinguish the tinnitus network from healthy individuals in different frequency bands through the sensor level EEG analysis. To detect the underlying neurophysiological differences, network analysis was performed based on WPLI functional connectivity, which is able to identify true variations in phase-synchronization and has less sensitivity to common noise sources [28].

Based on our findings, the significant differences in the functional connectivity between tinnitus and healthy groups were more dominant in the alpha band [39]. The WPLI connectivity declined in the alpha band for the tinnitus group, which supports the findings [40]. The reduction of functional connectivity in the alpha band could be due to reduced activity of alpha waves in the auditory cortex, predicted in Global Brain Model (GBM) of tinnitus [41].

In our study, the variations of connectivity patterns in the tinnitus group were observed within the frontal, temporal and parietal regions. Several EEG, MEG, and fMRI-based studies have reported non-auditory areas, especially the prefrontal cortex (PFC) are involved in tinnitus [10, 42, 43]. Specifically, an MEG study has reported that the tinnitus neural activity alterations are more dominant in the temporal, left frontal and right parietal regions [44]. Furthermore, the first evidence for changes of long-range synchronization in non-auditory areas was presented [45], which showed the association of right frontal and parietal lobes in tinnitus. It has also been reported that the connectivity pattern of chronic tinnitus is disrupted in PFC [46]. The authors [46] examined the tinnitus brain networks through the functional and effective connectivity analysis of fMRI data and deduced that PFC is the main integrative cortical hub, which plays the main role in the phantom sound perception in tinnitus. Accordingly, PFC should be consid- ered as a key region involved in tinnitus, when associated with both emotional and sensory factors of tinnitus [47]. These findings are all in consistent with our results, which identified the significant disruption of connectivity network in middle temporal (T7 corresponds to BA 21), supramarginal gyrus (CP5 corresponds to BA 40), frontopolar (FP1 corresponds to BA 10) and intermediate frontal $(\mathrm{Fz}$ corresponds to BA 08) regions.

The results of our graph theoretical analysis illustrated that the clustering coefficient and the local efficiency of tinnitus network showed an enhancement compared to controls in alpha2, beta2, and beta 3 frequency bands, while a significant reduction of these two measures could be observed in the delta, theta, and alphal frequency bands for the tinnitus group. On the other hand, the characteristic path length of tinnitus network significantly increased in the alpha2, beta2, and beta3 frequency bands and showed a reduction in theta frequency band. Thus, the tinnitus network indicated relatively stronger segregation and weaker integration in alpha2, beta2, and beta3 frequency bands. The results would be interpreted that the tinnitus network is more segregated than the healthy group in higher frequencies, while has the weaker global efficiency. Thus, high-frequency bands show that reduced small world attributes with the reduced integration, as we expected [38]. Furthermore, the tinnitus group presented lower segregation and greater integration relative to the healthy group in the theta frequency domain. This finding would be interpreted as the deviation of tinnitus network from the small world topology towards the randomness in the theta band.

Four-network measures applied as features to distinguish between tinnitus and healthy individuals led to the high sensitivity, specificity, and accuracy for all frequency bands. The highest accuracy, sensitivity or specificity of $100 \%$ was observed in several frequency bands. The lowest accuracy of 75\% was acquired in alpha 2 and beta 3 frequency 
bands. The smallest values for sensitivity and specificity were 66.7 in alpha 2 and beta3, respectively. In general, the classification results were relatively lower in the alpha 2 and beta 3 compared to other frequency bands. The best classification performance was achieved in the beta2 frequency band with accuracy, sensitivity, and specificity of $100 \%$.

As the potential limitation of the present study, sensor space analysis of the EEG data cannot specify the appropriate underlying neural activity of the brain. Although WPLI connectivity measure was employed to reduce the sensitivity to common noise sources, one can consider the possible noise effect in the results. Hence, it might be more desirable to employ the source-localized EEG for further connectivity and graph theory analysis in future studies. In addition, the limited number of subjects can be assumed as the main limitation of this study. Thus, the larger sample size would be suggested in future works. However, the lack of matching between two groups in terms of hearing levels was unavoidable due to the high prevalence of hearing loss in individuals with tinnitus.

\section{Conclusion}

The results demonstrate that four network measures i.e. the node strength, clustering coefficients, local efficiency and characteristic path length could successfully discriminate the tinnitus from the healthy normal group. The experiment supports the feasibility of quantifying brain networks to assess this specific pathophysiological characteristic of the brain in diagnostic/treatment procedures as our fundamental contribution.

\section{Acknowledgment}

This study is related to the Ph.D. thesis NO. 441 Shahid Beheshti University of Medical Sciences, Tehran, Iran. We also appreciate the "Student Research Committee" and "Research \& Technology Chancellor" in Shahid Beheshti University of Medical Sciences for their financial support of this study.

Authors would like to acknowledge Iranian National Brain Mapping Laboratory (NBML), Tehran, Iran, for providing data acquisition (analysis) service for this research work.

\section{Conflict of Interest}

None

\section{References}

1. Eggermont JJ. Central tinnitus. Auris Nasus Larynx. 2003;30:7-12.

2. Snow JB. Tinnitus: theory and management. PMPH-USA; 2004.

3. Møller AR, Langguth B, De Ridder D, Kleinjung T. Textbook of tinnitus. Springer Science \& Business Media; 2010.

4. Meyerhoff W, Cooper J. Tinnitus. Otolaryngology. 1991;2:1169-79.

5. Phoon WH, Lee HS, Chia SE. Tinnitus in noiseexposed workers. Occup Med (Lond). 1993;43:358. PubMed PMID: 8422445.

6. Eggermont JJ, Roberts LE. The neuroscience of tinnitus. Trends Neurosci. 2004;27:676-82.

7. Norena A, Micheyl C, Chery-Croze S, Collet L. Psychoacoustic characterization of the tinnitus spectrum: implications for the underlying mechanisms of tinnitus. Audiol Neurootol. 2002;7:358-69. doi: 10.1159/000066156. PubMed PMID: 12401967.

8. Elgoyhen AB, Langguth B, De Ridder D, Vanneste $S$. Tinnitus: perspectives from human neuroimaging. Nat Rev Neurosci. 2015;16:632-42. doi: 10.1038/nrn4003. PubMed PMID: 26373470.

9. Mohan A, De Ridder D, Vanneste S. Graph theoretical analysis of brain connectivity in phantom sound perception. Sci Rep. 2016;6:19683. doi: 10.1038/srep19683. PubMed PMID: 26830446; PubMed PMCID: PMC4735645.

10. Vanneste S, De Ridder D. The auditory and nonauditory brain areas involved in tinnitus. An emergent property of multiple parallel overlapping subnetworks. Front Syst Neurosci. 2012;6:31. doi: 10.3389/fnsys.2012.00031. PubMed PMID: 22586375; PubMed PMCID: PMC3347475.

11. Achard S, Salvador R, Whitcher B, Suckling J, Bullmore $\mathrm{E}$. A resilient, low-frequency, small-world human brain functional network with highly connected association cortical hubs. J Neurosci. 2006;26:6372. doi: 10.1523/JNEUROSCI.3874-05.2006. PubMed PMID: 16399673.

12. Bullmore E, Sporns 0. Complex brain networks: 
Tinnitus Detection based on Network Analysis

graph theoretical analysis of structural and functional systems. Nat Rev Neurosci. 2009;10:186-98. doi: 10.1038/nrn2575. PubMed PMID: 19190637.

13. Buzsaki G, Geisler C, Henze DA, Wang XJ. Interneuron Diversity series: Circuit complexity and axon wiring economy of cortical interneurons. Trends Neurosci. 2004;27:186-93. doi: 10.1016/j. tins.2004.02.007. PubMed PMID: 15046877.

14. Stam CJ, Reijneveld JC. Graph theoretical analysis of complex networks in the brain. Nonlinear Biomed Phys. 2007;1:3. doi: 10.1186/1753-46311-3. PubMed PMID: 17908336; PubMed PMCID: PMC1976403.

15. Sporns 0, Honey CJ. Small worlds inside big brains. Proc Natl Acad Sci U S A. 2006;103:1921920. doi: 10.1073/pnas.0609523103. PubMed PMID: 17159140; PubMed PMCID: PMC1748207.

16. Watts DJ, Strogatz SH. Collective dynamics of 'small-world' networks. Nature. 1998;393:440-2. doi: 10.1038/30918. PubMed PMID: 9623998.

17. Vertes PE, Alexander-Bloch AF, Gogtay N, Giedd JN, Rapoport JL, Bullmore ET. Simple models of human brain functional networks. Proc Natl Acad Sci U S A. 2012;109:5868-73. doi: 10.1073/ pnas.1111738109. PubMed PMID: 22467830; PubMed PMCID: PMC3326510.

18. Stam CJ. Modern network science of neurological disorders. Nat Rev Neurosci. 2014;15:683-95. doi: 10.1038/nrn3801. PubMed PMID: 25186238.

19. Mohan A, De Ridder D, Vanneste S. Graph theoretical analysis of brain connectivity in phantom sound perception. Sci Rep. 2016;6:19683. doi: 10.1038/srep19683. PubMed PMID: 26830446; PubMed PMCID: PMC4735645.

20. McCombe A, Baguley D, Coles R, McKenna L, McKinney $C$, Windle-Taylor $P$, et al. Guidelines for the grading of tinnitus severity: the results of a working group commissioned by the British Association of Otolaryngologists, Head and Neck Surgeons, 1999. Clin Otolaryngol Allied Sci. 2001;26:388-93. PubMed PMID: 11678946.

21. Delorme A, Makeig S. EEGLAB: an open source toolbox for analysis of single-trial EEG dynamics including independent component analysis. $J$ Neurosci Methods. 2004;134:9-21. doi: 10.1016/j. jneumeth.2003.10.009. PubMed PMID: 15102499.

22. Oostenveld R, Fries P, Maris E, Schoffelen JM. FieldTrip: Open source software for advanced analysis of MEG, EEG, and invasive electrophysiological data. Comput Intell Neurosci. 2011;2011:156869. doi: 10.1155/2011/156869. PubMed PMID: 21253357; PubMed PMCID: PMC3021840.

23. Fraga Gonzalez G, Van der Molen MJW, Zaric G,
Bonte M, Tijms J, Blomert L, et al. Graph analysis of EEG resting state functional networks in dyslexic readers. Clin Neurophysiol. 2016;127:3165-75. doi: 10.1016/j.clinph.2016.06.023. PubMed PMID: 27476025.

24. Hardmeier M, Hatz F, Bousleiman H, Schindler C, Stam CJ, Fuhr P. Reproducibility of functional connectivity and graph measures based on the phase lag index (PLI) and weighted phase lag index (wPLI) derived from high resolution EEG. PLOS One. 2014:9:e108648. doi: 10.1371/journal.pone.0108648. PubMed PMID: 25286380; PubMed PMCID: PMC4186758.

25. Rubinov M, Sporns 0. Complex network measures of brain connectivity: uses and interpretations. Neuroimage. 2010;52:1059-69. doi: 10.1016/j.neuroimage.2009.10.003. PubMed PMID: 19819337.

26. Vanneste S, Plazier M, Van Der Loo E, Van De Heyning $P$, De Ridder D. The difference between uni- and bilateral auditory phantom percept. Clin Neurophysiol. 2011;122:578-87. doi: 10.1016/j. clinph.2010.07.022. PubMed PMID: 20801079.

27. Vanneste S, Van De Heyning P, De Ridder D. The neural network of phantom sound changes over time: a comparison between recent-onset and chronic tinnitus patients. Eur J Neurosci. 2011;34:718-31. doi: 10.1111/j.1460-9568.2011.07793.x. PubMed PMID: 21848924.

28. Vinck M, Oostenveld $R$, Van Wingerden $M$, Battaglia F, Pennartz CM. An improved index of phasesynchronization for electrophysiological data in the presence of volume-conduction, noise and sample-size bias. Neuroimage. 2011;55:1548-65. doi: 10.1016/j.neuroimage.2011.01.055. PubMed PMID: 21276857.

29. Cohen MX. Analyzing neural time series data: theory and practice. London, England: MIT press; 2014.

30. Ewald A, Aristei S, Nolte G, Abdel Rahman R. Brain Oscillations and Functional Connectivity during Overt Language Production. Front Psychol. 2012;3:166. doi: 10.3389/fpsyg.2012.00166. PubMed PMID: 22701106; PubMed PMCID: PMC3369188.

31. Haufe S, Nikulin VV, Muller KR, Nolte G. A critical assessment of connectivity measures for EEG data: a simulation study. Neuroimage. 2013;64:120-33. doi: 10.1016/j.neuroimage.2012.09.036. PubMed PMID: 23006806.

32. Vapnik $V$. The nature of statistical learning theory. Springer science \& business media; 2013.

33. Burges CJ. A tutorial on support vector machines for pattern recognition. Data mining and knowl- 
edge discovery. 1998;2:121-67.

34. Alvar AA, Deevband MR, Ashtiyani M. Neutron spectrum unfolding using radial basis function neural networks. Appl Radiat Isot. 2017;129:3541. doi: 10.1016/j.apradiso.2017.07.048. PubMed PMID: 28802156.

35. Taherisadr M, Dehzangi 0, Parsaei H. Single Channel EEG Artifact Identification Using Two-Dimensional Multi-Resolution Analysis. Sensors (Basel). 2017;17(12): 2895 doi: 10.3390/s17122895. PubMed PMID: 29236042; PubMed PMCID: PMC5750748.

36. Latora V, Marchiori M. Efficient behavior of smallworld networks. Phys Rev Lett. 2001;87:198701. doi: 10.1103/PhysRevLett.87.198701. PubMed PMID: 11690461.

37. Humphries MD, Gurney K. Network 'smallworld-ness': a quantitative method for determining canonical network equivalence. PLOS One. 2008;3:e0002051. doi: 10.1371/journal. pone.0002051. PubMed PMID: 18446219; PubMed PMCID: PMC2323569.

38. Elgoyhen $A B$, Langguth $B$, Vanneste $S$, De Ridder D. Tinnitus: network pathophysiology-network pharmacology. Front Syst Neurosci. 2012;6:1. doi: 10.3389/fnsys.2012.00001. PubMed PMID: 22291622; PubMed PMCID: PMC3265967.

39. Zobay 0, Palmer AR, Hall DA, Sereda M, Adjamian $P$. Source space estimation of oscillatory power and brain connectivity in tinnitus. PLOS One. 2015;10:e120123. doi: 10.1371/journal.pone.0120123. PubMed PMID: 25799178; PubMed PMCID: PMC4370720.

40. Schlee W, Hartmann T, Langguth B, Weisz N. Abnormal resting-state cortical coupling in chronic tinnitus. BMC Neurosci. 2009;10:11. doi: 10.1186/1471-2202-10-11. PubMed PMID:
19228390; PubMed PMCID: PMC2649130.

41. Schlee W, Lorenz I, Hartmann T, Müller N, Schulz $\mathrm{H}$, Weisz N. A global brain model of tinnitus. Textbook of tinnitus. New York: Springer; 2011. p. 161-9.

42. De Ridder D, Vanneste $S$, Weisz N, Londero A, Schlee W, Elgoyhen $A B$, et al. An integrative model of auditory phantom perception: tinnitus as a unified percept of interacting separable subnetworks. Neurosci Biobehav Rev. 2014;44:16-32. doi: 10.1016/j.neubiorev.2013.03.021. PubMed PMID: 23597755.

43. Vanneste S, Plazier M, Der Loo E, De Heyning PV, Congedo M, De Ridder D. The neural correlates of tinnitus-related distress. Neuroimage. 2010;52:470-80. doi: 10.1016/j.neuroimage.2010.04.029. PubMed PMID: 20417285.

44. Weisz N, Moratti S, Meinzer M, Dohrmann K, Elbert $\mathrm{T}$. Tinnitus perception and distress is related to abnormal spontaneous brain activity as measured by magnetoencephalography. PLOS Med. 2005;2:e153. doi: 10.1371/journal.pmed.0020153. PubMed PMID: 15971936; PubMed PMCID: PMC1160568.

45. Schlee W. Towards a Global Model of Tinnitus Perception: Multiple Evidence for a Long-Range Cortical Tinnitus Network. Diss; 2009.

46. Chen YC, Feng Y, Xu JJ, Mao CN, Xia W, Ren J, et al. Disrupted Brain Functional Network Architecture in Chronic Tinnitus Patients. Front Aging Neurosci. 2016;8:174. doi: 10.3389/fnagi.2016.00174. PubMed PMID: 27458377; PubMed PMCID: PMC4937025.

47. Jastreboff PJ. Phantom auditory perception (tinnitus): mechanisms of generation and perception. Neurosci Res. 1990;8:221-54. PubMed PMID: 2175858. 